\title{
Genotype diversity of Mycobacterium isolates from children in Jimma, Ethiopia
}

Bereket Workalemahu ${ }^{1,2,3^{*}}$, Stefan Berg ${ }^{4}$, Wondewosen Tsegaye ${ }^{3}$, Alemseged Abdissa ${ }^{3}$, Tsinuel Girma ${ }^{5}$, Markos Abebe ${ }^{1}$ and Abraham Aseffa ${ }^{1}$

\begin{abstract}
Background: Paediatric tuberculosis (TB) is poorly addressed in Ethiopia and information about its magnitude and the genotype distribution of the causative Mycobacterium tuberculosis strains responsible for its spread are scanty.

Methods: Gastric lavage or sputum samples were collected from consecutively enrolled TB suspect children visiting Jimma University Hospital in 2011 and cultured on Middlebrook 7H11 and Löwenstein-Jensen media. Acid fast bacterial (AFB) isolates were subjected to molecular typing targeting regions of difference (RDs), 165 rDNA gene and the direct repeat (DR) region using multiplex polymerase chain reaction (mPCR), gene sequencing and spoligotyping, respectively. Molecular drug susceptibility testing of M. tuberculosis isolates was performed by Genotype ${ }^{\oplus}$ MTBDRplus line probe assay (LPA) (Hain Life Sciences, Germany).

Results: Gastric lavage $(n=43)$ or sputum $(n=58)$ samples were collected from 101 children and 31.7\% (32/101) of the samples were positive for AFB by microscopy, culture and/or PCR. Out of 25 AFB isolates, 60\% (15/25) were identified as M. tuberculosis by PCR, and $40 \%$ isolates (10/25) were confirmed to be non-tuberculous mycobacteria (NTM) by genus typing and 165 rDNA gene sequencing. Lineage classification assigned the $M$. tuberculosis strains into Euro-American (EUA, 66.7\%; 10/15), East-African-Indian (EAl; 2/15), East-Asian (EA; 1/15) and Indio-Oceanic $(\mathrm{IO} ; 1 / 15)$ lineages. Seven M. tuberculosis strains were new to the SpolDB4 database. All of the M. tuberculosis isolates were susceptible to isoniazid (INH) and rifampicin (RIF), except for one strain (of spoligotype SIT-149 or T3_ETH family) which had a mutation at the inhA locus which often confers resistance to INH (low level) and ethionamide.

Conclusions: Analysis of the genetic population structure of paediatric M. tuberculosis strains suggested similarity with that of adults, indicating an on-going and active transmission of $M$. tuberculosis from adults to children in Ethiopia. There were no multidrug-resistant TB (MDR-TB) strains among the isolates.
\end{abstract}

Keywords: Paediatric TB, Mycobacterium, Paediatric spoligotype, Gastric lavage

\section{Background}

Tuberculosis (TB) accounts for more than 10\% of hospital admissions and death in children in developing countries such as Ethiopia [1-3]. The high burden of TB among children is assumed to be due to the high prevalence of TB in adults, HIV co-infection, malnutrition and other conditions related to poverty [4].

Addressing childhood tuberculosis is important not only because of the morbidity and mortality burden in children but also because it is a public health measure of

\footnotetext{
* Correspondence: bekicho@gmail.com

'Armauer Hansen Research Institute (AHRI/ALERT), Addis Ababa, Ethiopia

${ }^{2}$ Medical Laboratory Technology Team, Arba Minch College of Health

Sciences, Arba Minch, Ethiopia

Full list of author information is available at the end of the article
}

recent transmission of $M$. tuberculosis and thus a sentinel indicator of the effectiveness of TB control programmes. TB infection in childhood impacts on future control strategies as a source of transmission decades later due to reactivation of latency [5]. Ethiopia is a TB high burden country (prevalence of $237 / 100,000$ people) with low case detection rates [6]. Infection among children is likely to be similarly high since children acquire the disease mainly from adults [2,7]. The actual magnitude of paediatric TB is unknown in the country and national reports have so far provided little information on its prevalence [8].

Molecular characterization of strains has allowed for the analysis and better understanding of transmission dynamics, genetic phylogeny, strain virulence and drug

\section{Biomed Central}

(c) 2013 Workalemahu et al.; licensee BioMed Central Ltd. This is an Open Access article distributed under the terms of the Creative Commons Attribution License (http://creativecommons.org/licenses/by/2.0), which permits unrestricted use, distribution, and reproduction in any medium, provided the original work is properly cited. 
resistance $[9,10]$. Spoligotyping is one of the widely used molecular methods for simultaneous detection and typing of M. tuberculosis complex bacteria and the identification of epidemiological links between patients [11], although it has less discriminatory power compared to the restriction fragment length polymorphism (RFLP) typing using insertion element IS6110 [12].

Based on large sequence polymorphism, six main lineages have been described within the $M$. tuberculosis complex (MTBC) affecting humans: Indio-oceanic (IO) (Lineage 1), East-Asian (EA) (Lineage 2), East-African-Indian (EAI) (Lineage 3), Euro-American (EUA) (Lineage 4), West African Lineage I (Lineage 5) and West African Lineage II (Lineage 6) [13]. However, the naming and the grouping systems vary according to the molecular marker and the method of typing used. For example the SpolDB4 system assigns the MTBC organisms into 62 clades/lineages based on the genome variability at the DR locus (e.g. EAI, Beijing, CAS, Haarlem, T, X, AFRI 1 and AFRI 2) [14]. Gagneux and Small [15] have reviewed the different terminology and the genetic markers used to assign the MTBC into different phylogeographic groupings. Once the genotype data are available, different online computer programs and databases can assist in suggesting and assigning of the MTBC strains into genetic groups using a set of mathematical rules and according to the molecular marker used [16-18].

This study aimed to identify the genetic diversity of Mycobacterium isolates responsible for paediatric TB in Jimma University Hospital in southwest Ethiopia and compare this with already described diversity among adult Ethiopians.

\section{Methods}

\section{Study population and area}

Jimma University Hospital is located $355 \mathrm{~km}$ southwest of Addis Ababa. The hospital has 300 beds and provides curative and preventive service for 300-400 patients per day at its outpatient department [19]. The Paediatric and Child Health Department of the hospital gives inpatient and outpatient services to children $(<15$ years of age) and provides medical care for more than 100 children daily in the outpatient (OPD) section. Children under 15 years of age who presented to the OPD with clinically suspected tuberculosis according to the national guideline based on sign symptom complex, chest X-ray and tuberculin skin test (TST) findings were consecutively recruited into this study [20] over a period of one year in 2011.

\section{Ethical clearance}

The study was approved by the Ethics Review Boards of Jimma University, AHRI/ALERT Ethical Review Committee (Ref No: P015/09), and the National Health Research
Ethics Review Committee (NERC) of the Ethiopian Ministry of Science and Technology (Ref No: RDHE/82-92/ 2010). Informed consent was obtained from parents or guardians and additional assent was obtained from children older than 12 years of age.

\section{Sample collection and processing}

Three sputum samples were collected from each child able to expectorate sputum. From younger children unable to provide sputum, gastric lavage samples were collected in the inpatient department on three consecutive mornings after an overnight fast. This was done by inserting a nasogastric tube into the stomach immediately after the patients woke up in the morning [21]. A minimum of $30 \mathrm{ml}$ gastric fluid was drawn into a $50 \mathrm{ml}$ syringe attached to the tube and transferred to a $50 \mathrm{ml}$ sterile plastic tube. If there was no aspirate, the stomach was irrigated with $50 \mathrm{ml}$ of sterile physiologic saline and aspirated back into the syringe. Within 30 minutes of sample collection, sodium bicarbonate (100 mg of sodium bicarbonate per 5 to $10 \mathrm{ml}$ of aspirate) was used to neutralize the sample before storage at $-20^{\circ} \mathrm{C}$ for a maximum of 2 months until transported on ice to the TB laboratory of the Armauer Hansen Research Institute where the samples were further stored at $-20^{\circ} \mathrm{C}$ and processed for culture within 2 weeks of receipt.

The digestion-decontamination procedure of all collected samples was based on the method developed by Kubica et al. [22-25]. Briefly, a fresh solution of digestant was prepared by adding $0.5 \mathrm{mg}$ of NALC powder to $100 \mathrm{ml}$ of sterile $\mathrm{NaOH}$ and tri-sodium citrate mixture (4\% $\mathrm{NaOH}$ and $2.9 \%$ tri-sodium citrate; Sigma). The final concentration of $\mathrm{NaOH}$ in the digestant was $2 \%$. The samples were decontaminated by adding an equal volume of fresh digestant in a $50 \mathrm{ml}$ sterile plastic tube for 15 minutes at room temperature, and neutralized by excess of phosphate buffered saline (PBS) pH 7.2 (Sigma, Germany). The sample was centrifuged for 15 minutes at $3200 \mathrm{RCF}$ in a cooling ultracentrifuge. The supernatant was then discarded aseptically and the pellet resuspended in $3 \mathrm{ml}$ sterile PBS buffer.

\section{Culture}

Fluka's TB base medium was used to prepare LöwensteinJensen (LJ) slants according to manufacturer instructions (Fluka Chemie GmbH, Switzerland). To support the growth of $M$. bovis, one of the media contained pyruvate (Sigma, Germany). Gruft's mycobacterial supplement (Fluka Chemie GmbH, Switzerland), which contained penicillin, nalidixic acid and ribonucleic acid, was aseptically added to all LJ media as supplement for the selective cultivation of mycobacteria according to the manufacturer's recommendation (Fluka Chemie $\mathrm{GmbH}$, Switzerland). Agar-base solid media were prepared 
using Difco ${ }^{\text {tm }}$ Middlebrook 7H11 Agar (Difco Laboratories, USA).

Decontaminated samples were each inoculated on four LJ (supplemented with either pyruvate or glycerol) and one $7 \mathrm{H} 11$ agar slants. Tubes were incubated at $37^{\circ} \mathrm{C}$ and inspected for growth daily for the first week and weekly thereafter for eight weeks. Suspected colonies were tested for AFB by Ziehl-Neelsen staining and positive isolates were characterized by molecular techniques described below.

\section{Species identification}

Isolates were heat killed by suspending 2-3 AFB colonies in $500 \mu \mathrm{l}$ distilled water in a test tube and heated at $95^{\circ} \mathrm{C}$ submerged in an ultrasonic water bath for 1 hour. A set of six primers were used in a multiplex PCR to determine isolates for genus Mycobacterium, $M$. tuberculosis complex, M. intracellulare and $M$. avium in a single PCR assay according to Wilton and Cousins [26]. Isolates were considered as non-tuberculous mycobacteria (NTM), if they were identified as mycobacteria that did not belong to the MTBC by this multiplex PCR method. These organisms were subjected to $16 \mathrm{~S}$ rDNA sequencing for species identification; the PCR method, amplification and detection conditions have been described previously [27]. For isolates identified as MTBC, an additional set of 3 primers targeting Region of Difference 9 (RD9) was performed to differentiate $M$. tuberculosis from other members of the MTBC.

\section{Spoligotyping}

MTBC organisms confirmed by RD9 typing were further characterized by spoligotyping. The procedure involved heat killing isolates, PCR amplification of spacers in the DR locus and detection according to previously described protocol [10]. M. tuberculosis H37Rv, M. bovis BCG and Qiagen RNAse free water was included as positive and negative controls. The spoligotyping data was analyzed using spolTools program [28].

\section{GenoType ${ }^{\oplus}$ MTBDRplus assay}

Sensitivity of the isolated $M$. tuberculosis strains to isoniazid (INH) and rifampicin (RIF) was tested with the GenoType ${ }^{\circ}$ MTBDRplus line probe assay (LPA) performed according to the manufacturer's protocol (Hain Life Science GmbH, Germany).

\section{Results}

\section{Characteristics of the study participants}

A total of 121 participants were included in this study and the male to female ratio was 1.3. The age of the patients ranged from one to fifteen with a mean age of 6.5 years. Forty three percent $(52 / 121)$ of the participants were under 5 years of age (Table 1). All of the
Table 1 Clinical and socio-demographic characteristics of consecutively enrolled childhood TB suspects visiting Jimma University Hospital, 2011 ( $n=121$ )

\begin{tabular}{|c|c|c|c|}
\hline Risk factor & Category & No & $\%$ \\
\hline \multirow[t]{3}{*}{ Age (in year) } & $1-5$ & 52 & 43 \\
\hline & $6-10$ & 46 & 38 \\
\hline & $11-15$ & 23 & 19 \\
\hline \multirow[t]{2}{*}{ Sex } & Male & 69 & 57 \\
\hline & Female & 52 & 43 \\
\hline \multirow[t]{2}{*}{ Contact history } & Yes & 67 & 55 \\
\hline & No & 54 & 45 \\
\hline \multirow[t]{2}{*}{ Fever } & Yes & 98 & 81 \\
\hline & No & 23 & 19 \\
\hline \multirow[t]{2}{*}{ Night sweats } & Yes & 103 & 85 \\
\hline & No & 18 & 15 \\
\hline \multirow[t]{2}{*}{ HIV } & Reactive & 15 & 12 \\
\hline & Non-reactive & 106 & 88 \\
\hline \multirow[t]{3}{*}{ Tuberculin skin test } & $0 \mathrm{~mm}$ & 65 & 54 \\
\hline & $1-10 \mathrm{~mm}$ & 34 & 28 \\
\hline & $>10 \mathrm{~mm}$ & 22 & 18 \\
\hline \multirow[t]{4}{*}{ Anthropometry } & Normal & 43 & 36 \\
\hline & Mild malnourished & 28 & 23 \\
\hline & Moderate malnourished & 29 & 24 \\
\hline & Severe malnourished & 21 & 17 \\
\hline
\end{tabular}

All participants in the study had cough of greater than 2 weeks of duration. The sign and symptom complex was defined as: night sweats, sweating that leads to wetting of the bed sheet; fever, body temperature of $>37.5^{\circ} \mathrm{C}$; close contact, living in the same household as, or in frequent contact with, a source case with sputum smear-positive pulmonary $\mathrm{TB}$ or clinically diagnosed TB; and anthropometry were based on weight, height/length and mid-upper arm circumference (MUAC) measurements and classification of acute malnutrition is according to Waterlow scheme (weight for height $(\mathrm{W} / \mathrm{H})$ (not malnourished, $\mathrm{W} / \mathrm{H}>90$ percent of reference median; mild malnutrition, W/H 80-90 percent of reference median; moderate acute malnutrition, W/H 70-80 percent of reference median; and severe acute malnutrition, $\mathrm{W} / \mathrm{H}<70$ percent of reference median).

participants had a clinical diagnosis of TB according to the national algorithm based on a combination of clinical and laboratory findings (including cough of $\geq 2$ weeks duration, contact history, tuberculin skin test positivity and chest X-ray). There was no sample for analysis from 20 participants $(16.5 \%)$ due to withdrawal or laboratory rejection of gastric lavage samples containing food particles. Among the 101 cases for whom samples were available, 58 (57.4\%) had provided sputum and 43 (42.6\%) gastric lavage.

\section{AFB microscopy and culture}

Two hundred and thirty samples from 101 patients (on average 2.3 samples from each child) were processed separately by culture and microscopy. Specimens from the same patient were considered together for analysis and a patient was considered positive when any one of 
the specimens from a patient was positive for mycobacteria. Smear microscopy was positive for AFB in 18/101 (17.8\%) and mycobacterial isolates were detected in 25/ $101(24.6 \%)$ patients. Gastric lavage was positive for AFB microscopy in 11 patients and culture yielded isolates in 10 cases (Figure 1).

\section{Molecular analysis}

The 25 AFB organisms isolated on culture were further characterized by molecular typing. Genus typing revealed that all AFB isolates belonged to the genus $\mathrm{Myco-}$ bacterium; 60\% (15/25) were isolates from the MTBC and the remaining strains $(10 / 25 ; 40 \%)$ were typed as NTM. 16S rDNA sequence analysis of the NTM isolates suggested $M$. fortuitum/M. porcinum, $M$. triviale, $M$. parascrofulaceum/M. seoulense/M. gastri/M. kansasii/M. nebraskense, M. fortuitum, M. terrae, M. engbackii and M. confluentis as the most likely species (Figure 2A). Further analysis of the MTBC strains identified all of them as $M$. tuberculosis, intact for the RD9 region (Figure 2B). Spoligotyping of these $M$. tuberculosis isolates showed that eight belonged to Shared International Type (SIT) number 37, 53, 149, 817, 289 and 120 according to the SpolDB4 database nomenclature [14], while the remaining 7 strains were new to this database. Using the Run TB-Lineage program, all (100\%) of the M. tuberculosis strains could be accurately categorized into one of the CDC's TB lineages: Euro-American (EUA, 66.7\%; 10/15), East-African-Indian (EAI; $2 / 15$ ) and East-Asian (EA; 1/15), and one strain
(PST-04) probably belonging to the Indio-Oceanic (IO; $1 /$ 15) lineage with a probability of 0.94 (Figure 3 ). All of the $M$. tuberculosis strains tested for drug resistance were found to be susceptible to INH and RIF except for one strain (of spoligotype SIT-149 or T3_ETH family) that had mutation at the inhA locus which often confers resistance to INH (low level) and ethionamide.

\section{Discussion}

The actual burden of childhood tuberculosis is unknown due to lack of routine case records and difficulty of diagnosing TB in children, both clinically and bacteriologically. However, it is estimated that in countries with high burden of TB up to $25 \%$ of the TB cases occur in children [6]. Thus, childhood tuberculosis represents a significant but still neglected clinical and public health problem [29]. Epidemiologically, investigation into paediatric TB is potentially more informative than adult $\mathrm{TB}$ in order to understand the status of recent transmission, evaluate effectiveness of TB control programmes or predict future trends in TB transmission [5]. Molecular tools such as spoligotyping have been standardized and widely used for species identification and depiction of the genetic diversity of MTBC organisms [10]. Using these tools, we characterized the clinical isolates of $M$. tuberculosis from paediatric patients in the Jimma area of south west Ethiopia and categorized them into previously established MTBC genetic groupings $[14,30]$ : 10 strains as belonging to Lineage 4 (EUA), 2 as Lineage 3 (EAI), 1 as Lineage 2 (EA), and 1 as

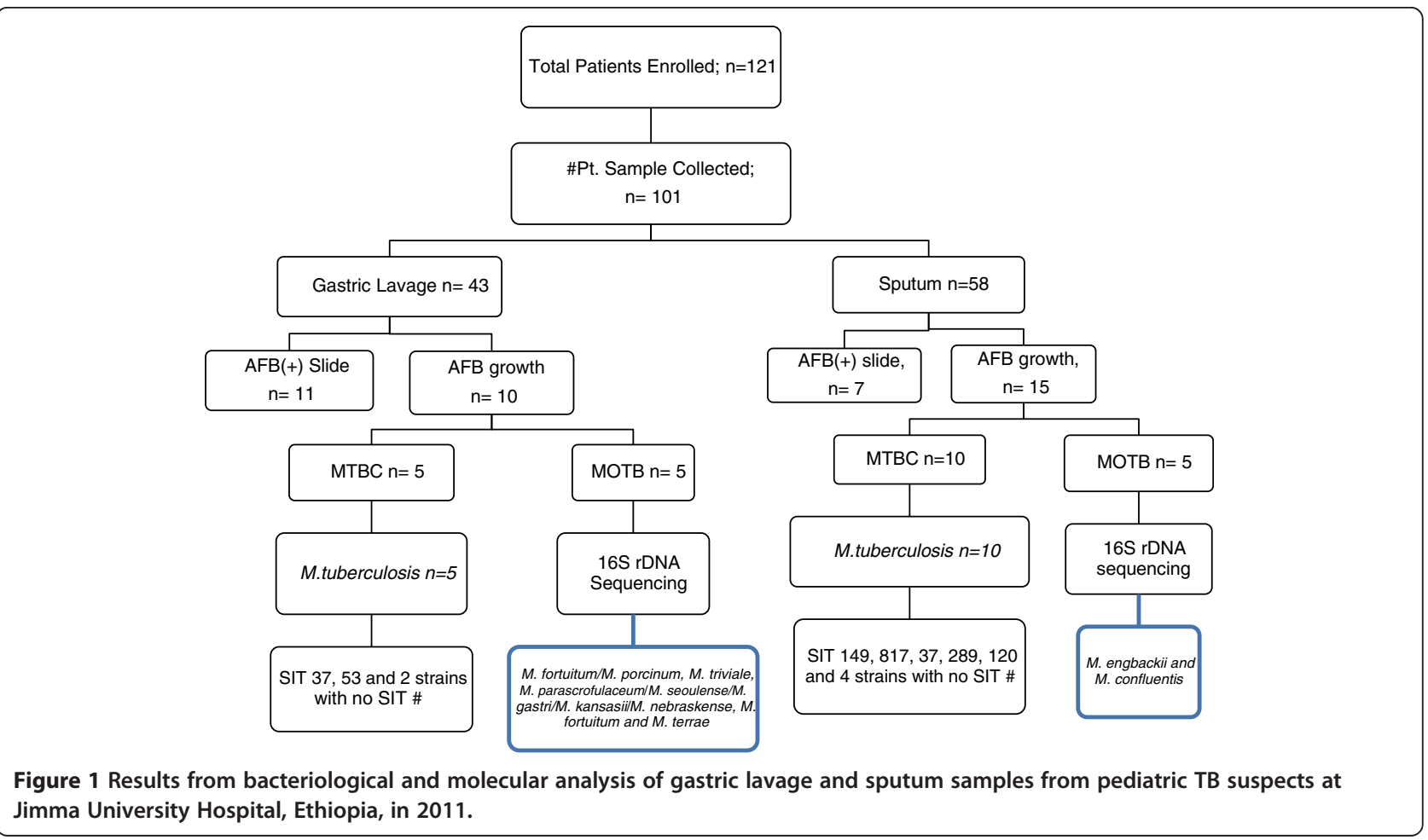




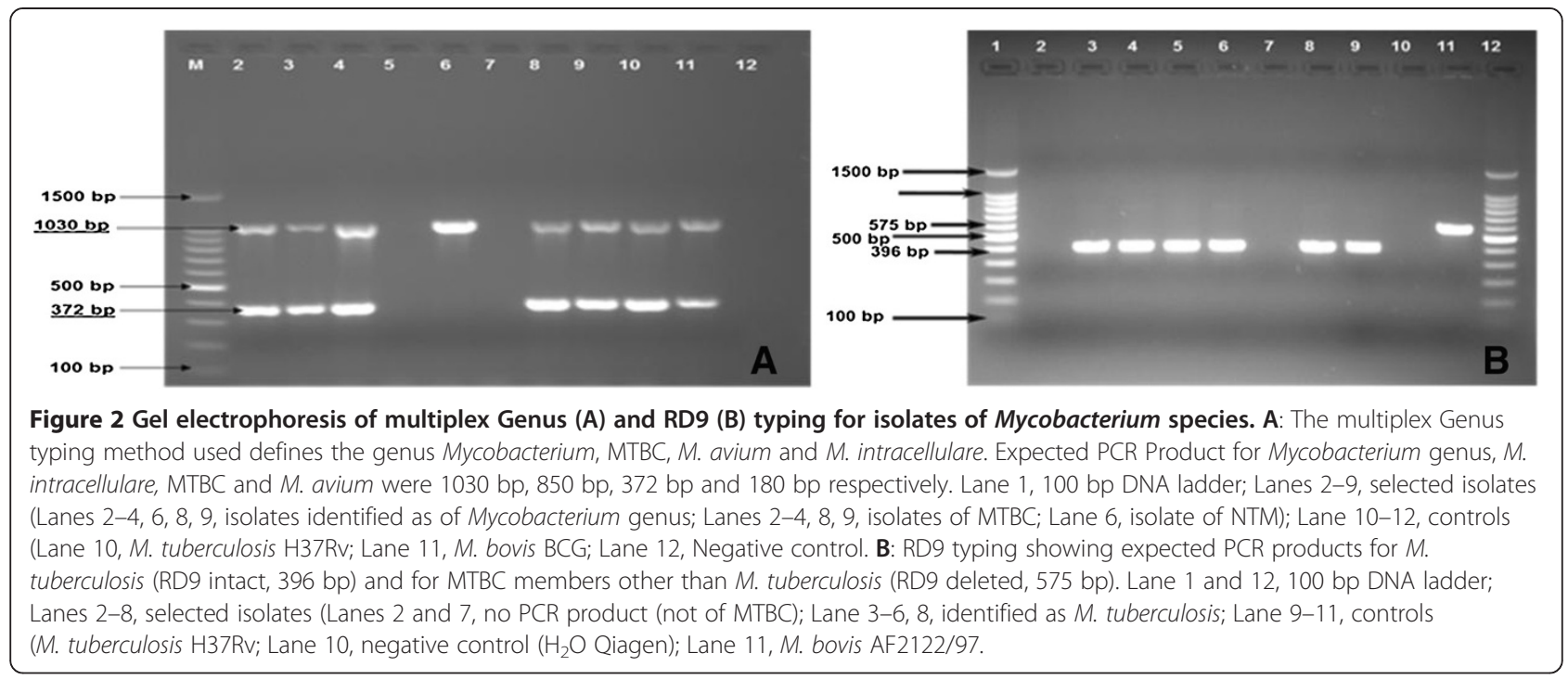

Lineage 1 (IO) according to the TB lineage program [16]. Interestingly, gastric lavage specimens were positive for AFB on smear microscopy at similar proportions as culture possibly due to limited survival of the bacilli in the acidic environment of gastric lavage.

It is well established that MTBC strains have a clonal genetic population structure and are highly restricted to geographical regions [15]. Firdessa et al. [31] reported the genetic population structure of the MTBC from an Ethiopian adult population that was predominantly composed of EUA (Lineage 4) and EAI (CAS, according to SpolDB4; Lineage 3). In agreement with that report, most of the strains isolated in this small series belonged to Lineage 4 (EUA) (60\%) and Lineage 3 or EAI/CAS (13\%). As far as can be concluded from the relatively few numbers and the single geographic area of recruitment, it seems that the population structure of our paediatric M. tuberculosis strains parallels that of the adult TB patients in Ethiopia. The contrary is possible as illustrated in a report on paediatric strains from Mexico,

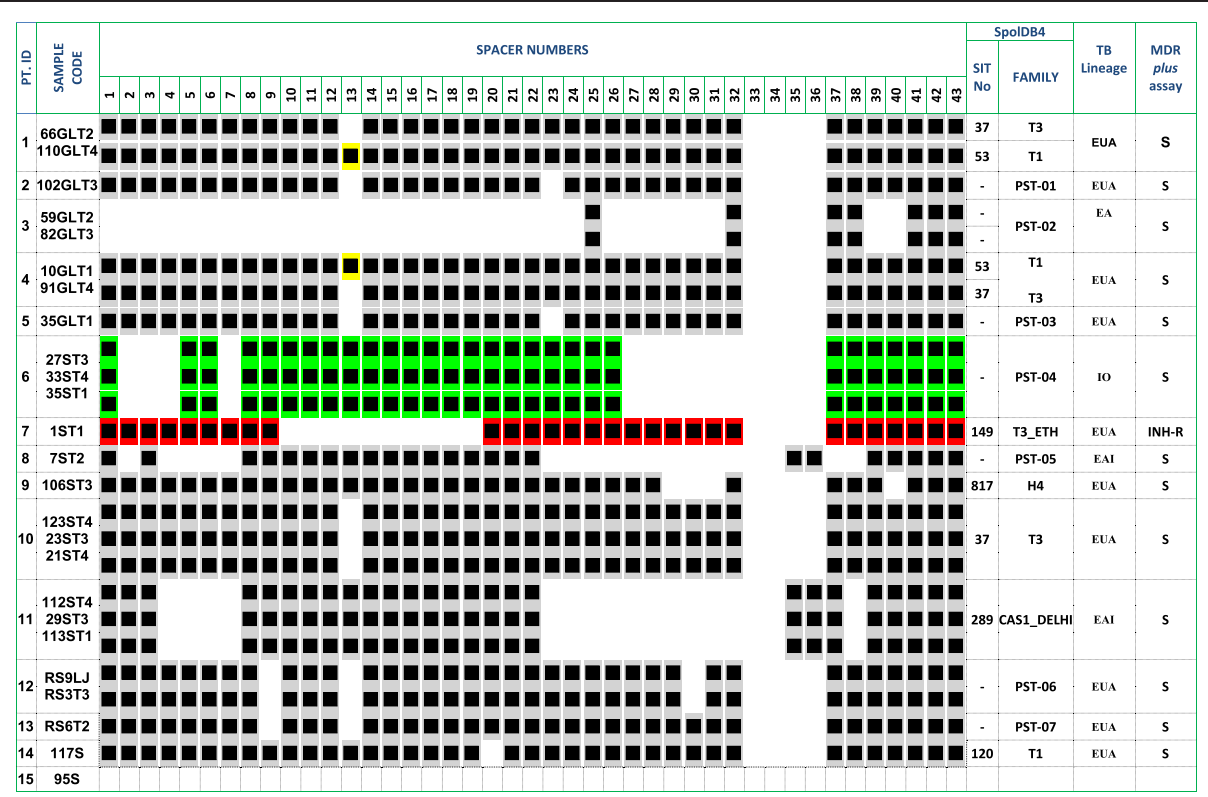

Figure 3 Spoligotype pattern of M. tuberculosis strains isolated from children with suspected TB in Jimma, Ethiopia, 2011. Spoligotype pattern of M. tuberculosis isolated from 14 children (one M. tuberculosis strain failed), also showing the corresponding SIT no, the family label (SPOLDB4) and SPOT CLUST grouping. H4, Haarlem; CAS1_DELHI, The Central Asian sub-lineage; EUA, Euro-American; EA, East-Asian; IO, Indo-Oceanic; EAl, East-African-Indian; PST, Orphan paediatric Spoligotype. 
which entirely differed from the one prevailing in adult TB patients in one particular state [32]. This fact suggests active transmission of $\mathrm{TB}$ from adults to children in Ethiopia, since children mainly acquire the disease from adults. Unlike our dataset, genotyping of isolates from 400 culture positive children in South Africa had identified the most contagious and virulent Beijing lineage to be the predominant strain in both paediatric [33] and adult South African patients [34].

High proportions $(46.7 \% ; 7 / 15)$ of the spoligotypes from our study children were orphan patterns, i.e., patterns not previously reported to the international database of SpolDB4 (SITVIT2 database) [14]. This finding suggests that the diversity of spoligotype patterns in Ethiopia is not fully explored. A report on Mexican children had also identified that up to $34.5 \%$ of the spoligotype patterns were orphan to the SITVIT2 database [32]. Construction of an MTBC strain database dedicated to paediatric isolates would be beneficial for research on TB in children. The clinical spectrum of disease and diagnostic approaches of childhood TB are different from those of adults and the two may need to be addressed separately [35].

Two thirds of the rapidly growing mycobacterial isolates from clinical specimens could cause true to probable infections and most of them could be isolated from respiratory sites. A large proportion of the patients with respiratory NTM isolates have underlying lung injuries and co-isolation of other pathogenic microorganisms such as the $M$. fortuitum complex group (M. fortuitum, M. porcium and others) is common [36]. The $M$. fortuitum group has also been isolated from respiratory samples of children in our study $(2 / 7 ; 28.5 \%)$. $M$. kansasii is a slow growing mycobacterium commonly found in tap water rather than in soil or salt water and consequently, M. kansasii related disease occurs in areas where contaminated drinking water is found [37]. Our M. kansasii isolate from gastric lavage could be a contaminant from the hospital tap water or an opportunistic nosocomial pathogen in children with a weakened resistance. An assessment of the mycobacteriology of the hospital environment might help clarify the source.

No multidrug-resistant TB (MDR-TB) strain was detected among the 25 paediatric TB isolates tested in this study. There was one INH-resistant strain (4\%). A study on adults in the same hospital in 2010 reported a $1.5 \%$ rate of multi-drug resistance and $13.4 \%$ INH resistance [38]. Although MDR TB appears to be rare among children at the site, the presence of MDR TB in the area albeit at relatively low proportions should alert health workers to the risk of MDR TB in children complicating care in an already difficult diagnostic environment.

\section{Conclusions}

Genetic typing of MTBC strains isolated from childhood TB patients in Jimma showed that the same lineages that dominate among adults, Lineages 4 and 3 (EUA and EAI or CAS), are prominent in children, suggesting on-going transmission of TB from adults to children in the area. A high proportion of strains were also unique to the SpolDB4 database and this calls for further studies to explore MTBC strains causing childhood TB in Ethiopia on a larger scale. To this end, constructing and populating a database dedicated to MTBC strains isolated from children is of potentially significant impact in understanding and monitoring the epidemiology of TB in children in this high burden country.

\section{Competing interests}

All the authors declare no competing interests.

\section{Authors' contributions}

BW was the primary researcher, conceived the study design, participated in sample collection, performed laboratory experiment, conducted the data analysis and drafted the manuscript for publication. SB performed part of the laboratory experiment, data analysis and draft manuscript. AAs, TG, WT, MA and $A A b$ participated in the design of the study and reviewed the initial and final drafts of the manuscript. All authors read and approved the final manuscript.

\section{Acknowledgements}

The study was funded by the Armauer Hansen Research Institute through its core support from Sida and NORAD. We would like to thank Jimma University for providing additional resources for conducting this research. We are also very grateful to Dr. Mesele Bezabeh, Dr. Zegeye Fanta, Dr. Wondim Getenet, Mr. Yewelsew Bekele, Mr. Eyasu Abegaz and Mr. Melaku Yohannes for their valuable support of the research process.

\section{Author details}

${ }^{1}$ Armauer Hansen Research Institute (AHRI/ALERT), Addis Ababa, Ethiopia. ${ }^{2}$ Medical Laboratory Technology Team, Arba Minch College of Health Sciences, Arba Minch, Ethiopia. ${ }^{3}$ Department of Laboratory Sciences and Pathology, Jimma University, Jimma, Ethiopia. ${ }^{4}$ Bovine TB Department, Animal Health and Veterinary Laboratories Agency (AHVLA), Weybridge, UK. ${ }^{5}$ Department of Paediatrics and Child Health, Jimma University, Jimma, Ethiopia.

Received: 15 February 2013 Accepted: 29 August 2013

Published: 4 September 2013

\section{References}

1. Kabra S, Lodha R, Seth V: Category base treatment of tuberculosis in children. Indian Pediatr 2004, 41:927-937.

2. Muluneh D, Shimelis D, Benti D: Analysis of admissions to the pediatric emergency ward of Tikur Anbessa Hospital in Addis Ababa, Ethiopia. Ethiop J Health Dev 2007, 21(1):48-52.

3. Ahmed T, Sobhan F, Ahmed S, Banu S, Mahmood A, Hyder K, Chisti M, Abdullah K, Mahfuz M, Abdus Salam M: Childhood tuberculosis: a review of epidemiology, diagnosis and management. Infect Dis J Pak 2008, 17(02):52-60.

4. Shamara S, Sarin R, Khalid U, Singla N, Shararma P, Behera D: The DOTS strategy for treatment of Paediatric Pulmonary TB in south Delhi, India. 2007. Int J Tuberc Lung Dis 2007, 11(12):74-80.

5. Starke JR: Pediatric tuberculosis: time for a new approach. Tuberculosis (Edinb) 2003, 83(1-3):208-212.

6. World Health Organization: Global tuberculosis report 2012. France: World Health Organization; 2012.

7. Glidey Y, Hable D: TB in children: an analysis of 412 cases. Ethiop Med 1983, 21:161-167. 
8. Federal Ministry of Health of Ethiopia: Tuberculosis Leprosy and TB/HIV Prevention and Control Programme Manual. 4th edition. Federal Ministry of Health Ethiopia; 2008.

9. Parsons LM, Brosch R, Cole ST, Somoskovi A, Loder A, Bretzel G, Van Soolingen D, Hale YM, Salfinger M: Rapid and simple approach for identification of Mycobacterium tuberculosis complex isolates by PCRbased genomic deletion analysis. J Clin Microbiol 2002, 40(7):2339-2345.

10. Kamerbeek J, Schouls L, Kolk A, van Agterveld M, van Soolingen D, Kuijper S, Bunschoten A, Molhuizen H, Shaw R, Goyal M, et al: Simultaneous detection and strain differentiation of Mycobacterium tuberculosis for diagnosis and epidemiology. J Clin Microbiol 1997, 35(4):907-914.

11. Gori A, Bandera A, Marchetti G, Degli Esposti A, Catozzi L, Nardi GP, Gazzola L, Ferrario G, van Embden JD, van Soolingen D, et al: Spoligotyping and Mycobacterium tuberculosis. Emerg Infect Dis 2005, 11(8):1242-1248.

12. van der Zanden AG, Kremer K, Schouls LM, Caimi K, Cataldi A, Hulleman A, Nagelkerke NJ, van Soolingen D: Improvement of differentiation and interpretability of spoligotyping for Mycobacterium tuberculosis complex isolates by introduction of new spacer oligonucleotides. J Clin Microbiol 2002, 40(12):4628-4639.

13. Gagneux S, DeRiemer K, Van T, Kato-Maeda M, de Jong BC, Narayanan S, Nicol M, Niemann S, Kremer K, Gutierrez MC, et al: Variable host-pathogen compatibility in Mycobacterium tuberculosis. Proc Natl Acad Sci USA 2006, 103(8):2869-2873.

14. Brudey K, Driscoll JR, Rigouts L, Prodinger WM, Gori A, Al-Hajoj SA, Allix C, Aristimuno L, Arora J, Baumanis V, et al: Mycobacterium tuberculosis complex genetic diversity: mining the fourth international spoligotyping database (SpolDB4) for classification, population genetics and epidemiology. BMC microbiology 2006, 6:23.

15. Gagneux S, Small PM: Global phylogeography of Mycobacterium tuberculosis and implications for tuberculosis product development. Lancet Infect Dis 2007, 7(5):328-337.

16. Aminian M, Shabbeer A, Bennett KP: A conformal Bayesian network for classification of Mycobacterium tuberculosis complex lineages. BMC Bioinform 2010, 11(3):S4

17. Shabbeer A, Ozcaglar C, Yener B, Bennett KP: Web tools for molecular epidemiology of tuberculosis. Infect Genet Evol 2012, 12(4):767-781.

18. Vitol I, Driscoll J, Kreiswirth B, Kurepina N, Bennett KP: Identifying Mycobacterium tuberculosis complex strain families using spoligotypes. Infect Genet Evol 2006, 6(6):491-504.

19. Beyene $G$, Haile-Amlak A: Antimicrobial sensitivity pattern of Campylobacter species among children in Jimma University Specialized Hospital, Southwest Ethiopia. Ethiop J Health Dev 2004, 18(3):185-189.

20. Federal Ministry of Health of Ethiopia: Guidelines for Paediatric HIV/AIDS Care and Treatment in Ethiopia. Federal Ministry of Health; 2007.

21. Palme I, Gudetta B, Bruchfeld J, Eriksson M, Giesecke J: Detection of Mycobacterium tuberculosis in gastric aspirate and sputum collected from Ethiopian HIV-positive and HIV-negative children in a mixed inand outpatient setting. Acta Paediatr 2004, 93:311-315.

22. Lobato M, Loeffler A, Furst $K$, Cole B, Hopewell P: Detection of Mycobacterium tuberculosis in gastric aspirates collected from children: hospitalization is not necessary. Pediatrics 1998, 102(4):e40

23. Winn JW, Allen S, Janda W, Koneman E, Procop G, Schreckenberger P: Koneman's Color atlas and text book of diagnostic microbiology. 6th edition. Baltimore, MD: Lippincott Williams \& Wilkins; 2006.

24. Blair BE, Weiser OL, Tull HA: Mycobacteriology Laboratory Methods. Denver, Colorado: U.S. Army Medical Research \& Nutrition Laboratory, Fitzsimons General Hospital; 1969.

25. Kubica GP, Gross WM, Hawkins JE, Sommers HM, Vestal AL, Wayne LG: Laboratory services for mycobacterial diseases. Am Rev Respir Dis 1975, 112(6):773-787.

26. Wilton S, Cousins D: Detection and identification of multiple mycobacterial pathogens by DNA amplification in a single tube. PCR Meth Appl 1992, 1(4):269-273.

27. Berg S, Firdessa R, Habtamu M, Gadisa E, Mengistu A, Yamuah L, Ameni G, Vordermeier M, Robertson BD, Smith NH, et al: The burden of mycobacterial disease in ethiopian cattle: implications for public health. PloS One 2009, 4(4):e5068.

28. Tang C, Reyes JF, Luciani F, Francis AR, Tanaka MM: spolTools: online utilities for analyzing spoligotypes of the Mycobacterium tuberculosis complex. Bioinformatics (Oxford, England) 2008, 24(20):2414-2415.
29. Newton SM, Brent AJ, Anderson S, Whittaker E, Kampmann B: Paediatric tuberculosis. Lancet Infect Dis 2008, 8(8):498-510.

30. Comas I, Homolka S, Niemann S, Gagneux S: Genotyping of genetically monomorphic bacteria: DNA sequencing in Mycobacterium tuberculosis highlights the limitations of current methodologies. PloS One 2009, 4(11):e7815

31. Firdessa R, Berg S, Hailu E, Schelling E, Gumi B, Erenso G, Gadisa E, Kiros T, Habtamu M, Hussein J, et al: Mycobacterial lineages causing pulmonary and extrapulmonary tuberculosis, ethiopia. Emerg Infect Dis 2013, 19(3):460-463

32. Macias Parra M, Kumate Rodriguez J, Arredondo Garcia JL, Lopez-Vidal Y, Castanon-Arreola M, Balandrano S, Rastogi N, Gutierrez Castrellon P: Mycobacterium tuberculosis complex genotype diversity and drug resistance profiles in a pediatric population in Mexico. Tuberc Res Treat 2011, 2011:239042

33. Hesseling: Pediatric tuberculosis: clinical and epidemiological reflections from a highly endemic setting. BMC Proc 2010, 4(3):012

34. Stavrum R, Mphahlele M, Ovreas K, Muthivhi T, Fourie PB, Weyer K, Grewal HM: High diversity of Mycobacterium tuberculosis genotypes in South Africa and preponderance of mixed infections among ST53 isolates. J Clin Microbiol 2009, 47(6):1848-1856.

35. Cuevas LE, Browning R, Bossuyt P, Casenghi M, Cotton MF, Cruz AT, Dodd LE, Drobniewski F, Gale M, Graham SM, et al: Evaluation of tuberculosis diagnostics in children: 2. Methodological issues for conducting and reporting research evaluations of tuberculosis diagnostics for intrathoracic tuberculosis in children. Consensus from an expert panel. J Infect Dis 2012, 205(2):S209-S215.

36. Han X, De I, Jackobson K: Rapidly growing mycobacteria: clinical and microbiological studies of 115 cases. Am J Clin Pathol 2007, 128:612-621.

37. Garcia Garcia JM, Palacios Gutierrez JJ, Sanchez Antuna AA: Respiratory infections caused by environmental mycobacteria. Arch Bronconeumol 2005, 41(4):206-219.

38. Abebe G, Abdissa K, Abdissa A, Apers L, Agonafir M, de-Jong BC, Colebunders $R$ : Relatively low primary drug resistant tuberculosis in southwestern Ethiopia. BMC Res Notes 2012, 5:225.

doi:10.1186/1756-0500-6-352

Cite this article as: Workalemahu et al:: Genotype diversity of Mycobacterium isolates from children in Jimma, Ethiopia. BMC Research Notes 2013 6:352

\section{Submit your next manuscript to BioMed Central and take full advantage of:}

- Convenient online submission

- Thorough peer review

- No space constraints or color figure charges

- Immediate publication on acceptance

- Inclusion in PubMed, CAS, Scopus and Google Scholar

- Research which is freely available for redistribution 